\title{
Effect of particle size ratio and contribution of particle size fractions on micromechanics of uniaxially compressed binary sphere mixtures
}

\author{
J. Wiącek ${ }^{1}$ - P. Parafiniuk ${ }^{1}$ - M. Stasiak ${ }^{1}$
}

Received: 10 August 2016 / Published online: 11 April 2017

(C) The Author(s) 2017. This article is an open access publication

\begin{abstract}
This paper is an extension of the recent work of Wiącek (Granul Matter 18:42, 2016), wherein geometrical parameters of binary granular mixtures with various particle size ratio and contribution of the particle size fractions were investigated. In this study, a micromechanics of binary mixtures with various ratio of the diameter of small and large spheres and contribution of small particles was analyzed using discrete element simulations of confined uniaxial compression. The study addressed contact normal orientation distributions, global and partial contact force distributions and pressure distribution in packings of frictional spheres. Additionally, the effect of particle size ratio and contribution of particle size fractions on energy dissipation in granular mixtures was investigated. The particle size ratio in binary packings was chosen to prevent small particles from percolating through bedding. The bimodality of mixtures was found to have a strong effect on distribution of contact normal orientation and distribution of normal contact forces in binary mixtures. Stress transfer in binary packing was also determined by both, particle size ratio and volume fraction of small particles. Dissipation of energy was higher in mixtures with higher particle size ratios and decreased with increasing contribution of small spheres in system.
\end{abstract}

Keywords Micromechanics · Discrete element method . Binary granular material $\cdot$ Particle size ratio

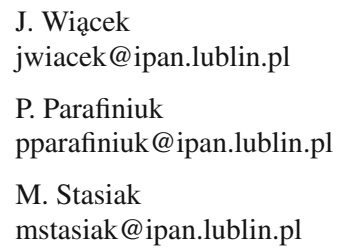

J. Wiącek

jwiacek@ipan.lublin.pl

P. Parafiniuk

pparafiniuk@ipan.lublin.pl

M. Stasiak

mstasiak@ipan.lublin.pl

1 Institute of Agrophysics, Polish Academy of Sciences, Doświadczalna 4, 20-290 Lublin, Poland

\section{Introduction}

Granular materials play important role in many industries, especially in agriculture and food industry, pharmaceutical, cosmetics, metallurgy, and building industries. Materials are subjected to technological processes whose design requires advanced research into granular matter. Although a number of studies has been conducted on granular materials over last few decades, providing crucial knowledge on properties of particulate solids and valuable insight into nature of interactions between granules, many phenomena related to complex behavior of granular assemblies still remain unexplained. Therefore, structural and mechanical properties of materials which determine production, handling processes and processing of particulate solids remain a focus of intense research in physics, chemistry and environmental science.

Most particle packings involved in industrial and natural processes consists of the common property of polydispersity. The degree of particle size heterogeneity determines a rearrangement of particles and contact network in granular system. It provides different compaction characteristics of particulate materials and powders, which is very important in, inter alia, powder technology, powder metallurgy, ceramics, chemical industry and industry of pharmaceutical tablet manufacturing. Granular packings may be composed of one, two, three or more particle size fractions. The more number of particle size fractions is, the more difficult interpretation of effects observed in granular material is. Therefore, the in-depth insight into a nature of simple particulate systems is required to understand behavior of more complex packings of non-uniformly sized grains. Binary mixtures represent the simplest case of polydisperse particulate systems. Up to date, a number of experimental $[2,3]$, theoretical [3-5] and numerical $[1,5,6]$ studies have been conducted to 
investigate structural and mechanical properties of bimodal particulate beds. It was found that packing density was sensitive to friction [7-9], particle size ratio [1,2,6,7,9-11] and contribution of particle size fractions in grain assembly [1$3,6,8,9,11]$. These factors were also found to determine geometric anisotropy, contact network and stress transmission in particulate system [9]. The studies conducted by McGeary [2] and Rassously [4] for binary mixtures with various particle size ratios have shown an increase in packing density of mixtures with volume fraction of small particles in packings increasing up to $60 \%$. A further increase in contribution of small particles in mixture resulted in decrease in packing density of samples. Packing density was larger in samples with smaller ratio between diameter of small and large spheres. Wiacek [1] indicated that, packing density of binary mixtures with small to large particle size ratios larger than 0.7 , did not reach maximum. It remained constant regardless on volume fraction of different fractions in mixtures. The experimental study conducted by Piston et al. [12] and DEM simulations prepared by Sánchez et al. [9] and by Wiacek [1] for bimodal mixtures indicated that both, particle size ratio (being a geometric factor) and contribution of particle size fractions in mixture (being a statistical factor) determined a partial coordination number. An increase in contribution of one of the size fraction in sample strongly affected number of contacts of different types and the larger spheres had more contacts with surrounding particles. Particle size ratio was found to have strong influence on anisotropy in the contact distribution and distribution of stress within the sample. Lade et al. [13] suggested that as the diameter ratio of small to large sphere decreased, the smaller granules better fitted within the pores between large particles resulting in reduced contribution of small grains in stress transfer. A numerical study conducted by Shire et al. [14] for bimodal packings of spheres have shown that small grains contributed approximately equally to stress transfer in packings with volume fraction of small particles not smaller than $30 \%$. In packings comprising smaller number of small grains, a smaller contribution of these grains to stress transfer was observed, which decreased with decreasing particle size ratio. As the small particles are able to fit more efficiently in the voids, they are less likely to interact with the large spheres and participate in forming strong force chains.

The interactions between particles in granular packing are the sources of potential energy that propagates throughout the contact network. Part of that energy dissipates in the plastic deformation and due to work of frictional or adhesive forces in contacts $[15,16]$. Interactions between grains in polydisperse granular packing are strongly affected by geometric and statistical factors. Therefore, dissipation of energy in particulate assembly is also determined by these factors $[17,18]$. Two-dimensional simulations of gravity-free Cou- ette flow of binary granular systems, conducted by Karion and Hunt [18], indicated increased rate of energy dissipation in mixtures when the ratio of small to large granules decreased. Energy dissipated in the collisions was proportional to the mass of the colliding bodies, resulting in greater energy dissipation in systems composed of larger granules. DEM simulations of uniaxial compression of polydisperse sphere packings, conducted by Wiacek and Molenda [19], have shown that a loss energy increased as the degree of particle size heterogeneity increased. It is well known that efficiency of industrial processes involving handling of granular materials significantly depends on dissipation of energy in system. Although a dissipative nature of forces acting on interacting particles is nowadays well-known, the search for method for reduction of energy loss during technological processes is still required. More insight is necessary to understand mechanisms of stress transfer and energy dissipation in non-uniformly sized particulate systems as well as to predict behavior of grain assembly under specific load conditions.

The review of literature shows that a large number of investigations involving binary granular mixtures addressed the ones with high difference between diameters of particles. Small grains percolated downwards through these beddings, which resulted in particle-size segregation affecting structural and mechanical properties of particulate assemblies. A reported project was devoted to the investigation of bimodal mixtures with relatively high particle size ratios, wherein percolation of small particles through larger ones did not take place. An influence of ratio between diameters of particles and contribution of granulometric fractions on mechanical properties of that kind of binary granular packings remains open question and is still insufficiently analyzed. Therefore, in this study, contact normal orientation distributions, global and partial contact force distributions and stress transfer in bimodal mixtures has been largely studied. Additionally, an effect of geometric and statistical factors on energy dissipation in particulate systems was investigated, which is of great interest in many industrial processes. As the experimental methods available do not provide complete information on interactions between particles inside the bedding, computational techniques find wide application. In the reported project, a Discrete Element Method was used to study mechanics of binary mixtures of spherical frictional particles.

\section{Materials and methods}

The numerical method applied in this paper is a Discrete Element Method (DEM). Discrete Element Method is a numerical technique for detailed investigation of mechanical 
behavior of granular systems, originally proposed by Cundall and Strack [20]. It allows for establishment of positions and velocities of particles in system through time integration of the ordinary differential equations formed for each individual particle on the basis of Newton's second law of motion.

The present study focuses on monodisperse and bidisperse sphere packings composed of the frictional cohesionless spheres with diameters of $8,6.35,4.762$ and $3.175 \mathrm{~mm}$. Binary mixtures were described by particle size ratio $g$, defined as a ratio between the diameter of small and large particles, and volume fraction of small spheres $(f)$, defined as a ratio between the volume of small particles and the volume of all particles in bedding. Bidisperse mixtures with particle size ratio of approximately $0.4,0.6$ and 0.8 , and volume fraction ranging between 0 and 1 were prepared. In each binary assembly, one of the fractions comprised spheres of the diameter of $8 \mathrm{~mm}$ and the second fraction was composed of particles with the diameter of $6.35,4.762$ or $3.175 \mathrm{~mm}$. In this study, particle size ratio was chosen larger than 0.4 to prevent small particles from percolating through bedding. The DEM input parameters, corresponding to the mechanical parameters of steel rods and steel walls are summarized in Table 1.

Three-dimensional simulations of the uniaxial confined compression test were performed by using EDEM software [21]. Samples were placed in a chamber of rectangular crosssection with rigid and frictional walls which did not deform under the applied load. The dimensions of sample were larger than 15 particle mean diameters which were adopted to be a representative elementary volume for polydisperse mixtures [22]. Granular material was compressed through the top platen that moved vertically with a constant velocity of $3 \mathrm{~m} / \mathrm{min}$ until a maximum vertical pressure on the uppermost spheres of $100 \mathrm{kPa}$ was adopted. The test procedure followed recommendations of the Eurocode 1 design standard [23]. Further details of contact model and generation procedure of sample are available in an earlier study by Wiaccek [1].

Table 1 DEM input parameters

\begin{tabular}{lll}
\hline Parameter & Steel & \\
\hline Poisson's ratio & 0.3 & \\
Shear modulus (GPa) & 77 & \\
Density $\left(\mathrm{kg} / \mathrm{m}^{3}\right)$ & 7804 & \\
Coefficient of restitution & Particle-particle & Particle-wall \\
& 0.4 & 0.4 \\
Coefficient of static friction & Particle-particle & Particle-wall \\
& 0.321 & 0.216 \\
Coefficient of rolling friction & Particle-particle & Particle-wall \\
& 0.01 & 0.01 \\
\hline
\end{tabular}

\section{Results and discussion}

\subsection{Packing microstructure}

In this section, effect of particle size ratio and contribution of granulometric fractions on structural properties of bidisperse mixtures subjected to a vertical pressure of $100 \mathrm{kPa}$ was studied. For this purpose, the evolution of solid fraction $(\Phi)$ of samples with various particle size ratios, defined as the fraction of sample volume filled by grains, with volume fraction of small particles was presented in Fig. 1. The mean values are plotted with the error bars indicating \pm one standard deviation. Solid fraction was significantly larger in binary mixtures with size ratio of 0.4 and 0.6 , as compared to monodisperse packings composed of large spheres. In these mixtures, an increase in solid fraction with an increase in $f$ value up to 0.6 was observed, which was then followed by decrease in $\Phi$ value with increasing contribution of small spheres in sample. A maximum $\Phi$ value was observed in packings with volume fraction of small particles of 0.6 , which corroborated findings reported earlier by inter alia McGeary [2], Rassously [3], Jalali and Li [6]. In samples with particle size ratio of 0.8 , solid fraction varied slightly with increasing contribution of small particles in mixtures. No evident maximum in value of parameter was observed in these packings, which has been reported earlier by Wiącek [1]. The author indicated that solid fraction did not reach maximum in binary samples wherein the ratio of the diameter of small and large spheres was larger than certain critical value.

In this study, an effect of geometric and statistical factors on geometric anisotropy of binary mixtures was investigated through the comparison of contact normal orientation distributions. A contact angle is defined as:

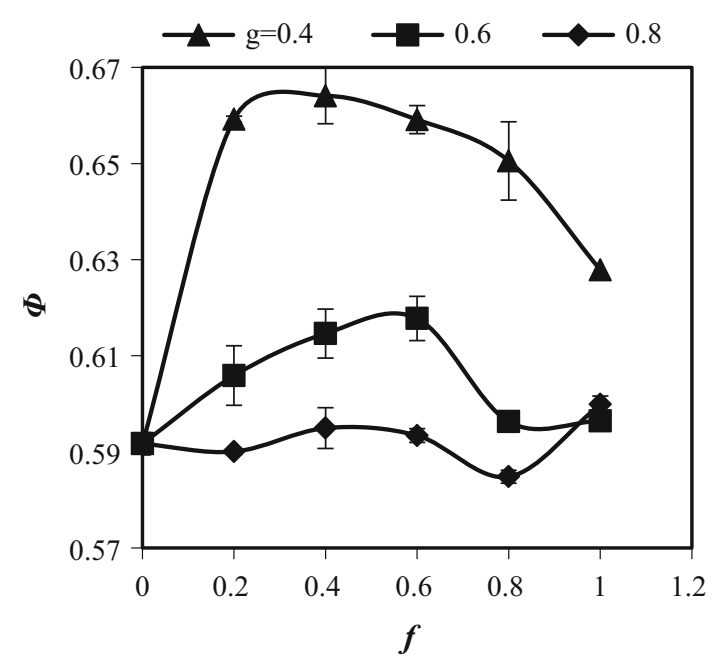

Fig. 1 Evolution of solid fraction versus volume fraction of small spheres in binary mixtures with various size ratios when subjected to a vertical load of $100 \mathrm{kPa}$ 
Fig. 2 Global distributions of contact normal orientation in mixtures with particle size ratio of 0.8 (a) and 0.4 (b) and volume fraction of small spheres of $40 \%$ ( $f=0.4)$, under compressive load of $100 \mathrm{kPa}$

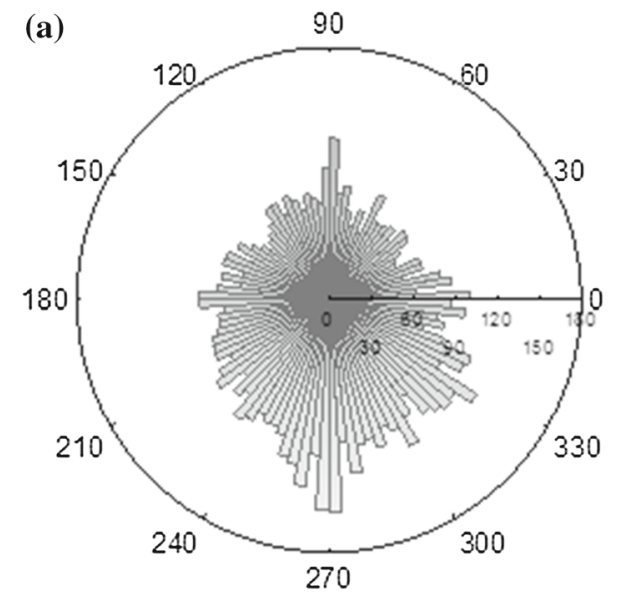

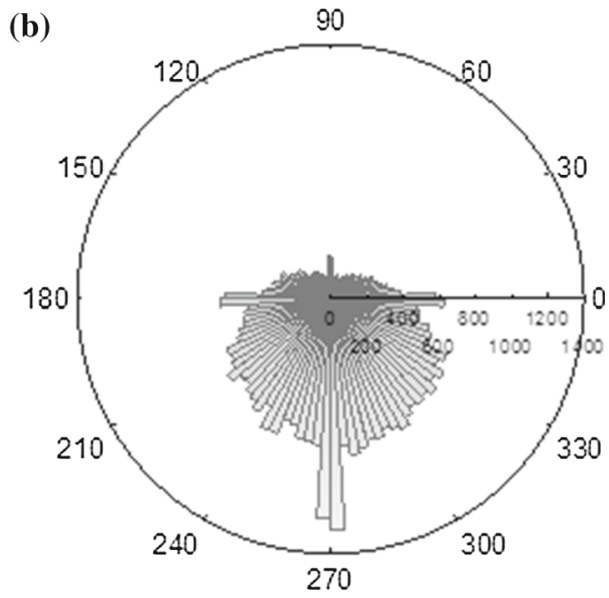

$\alpha=\arctan \frac{F_{z}^{n}}{F_{x}^{n}}$,

where $F_{z}^{n}$ and $F_{x}^{n}$ are the $z$ - and $x$-components of the contact normal force. Figure 2 presents distributions of contact normal orientation in compressed mixtures with various size ratios. Regardless on $g$ value, heterogeneous distribution of contact angles with a favored vertical contact direction was observed in examined packings. An increase in global anisotropy of the contact normal orientation was observed with increasing difference between the dimeters of small and large spheres in samples. Packings composed of particles with a small degree of particle size heterogeneity are arranged in a nearly crystalline formation with a favored vertical contact direction of $90^{\circ}$ and $270^{\circ}$. In samples with $g=0.8$, each particle is supported by several neighboring particles and it supports the other ones, providing more homogeneous distribution of contact normal orientations, as compared to mixtures with $g=0.4$. In packings with small $g$ value, number of contacts directed upward is smaller than number of contacts directed downward, resulting in asymmetric distribution. In more heterogeneous packings, where small grains partially fill the pores between larger particles and they do not necessarily support particles located higher, number of contacts ranging from $0^{\circ}$ to $180^{\circ}$ significantly decreases. In these mixtures, contacts directed downward prevail with favored contact direction of $270^{\circ}$. In the earlier study conducted by Wiacek and Molenda [17] for polydisperse granular mixtures with continuous particle size distribution, the authors observed more homogeneous distribution of contact force orientations in polydisperse packings as compared to monodisperse ones. These findings indicate that influence of polydispersity of granular packing on distribution of contact angles in sample is determined by a number of granulometric fractions. The authors suggest a presence of a certain number of granulometric fractions in particulate assembly above which anisotropy of the contact normal orientation decreases with increasing degree of particle size heterogeneity. An establishment of that number requires further study.

The partial distributions of contact normal orientation for large (11), large and small (ls) and small (ss) particles are shown in Fig. 3. In samples with $g=0.8$, a slight anisotropy in contacts between large spheres was observed, which was not visible in packings with smaller $g$ values. Regardless on $g$ value, no anisotropy was revealed by contact normal orientations between large and small spheres. In turn, for contacts between small spheres, a favored vertical contact direction was observed. In packings with $g=0.4$, contacts directed downward prevailed. An increase in difference between diameters of particles in mixtures resulted in more asymmetric distribution of the contact normal orientation with greater spread downward in frequency than upward.

These results were in agreement with findings reported by Sánchez et al. [9], who observed smaller partial anisotropy in the contact distribution in samples with smaller ratio between the small and large sphere. These authors also reported no relationship between anisotropy and contribution of particle size fractions in mixture. In this study, that issue was investigated in detail, providing results opposite to ones presented by Sánchez et al. [9]. Figure 4 presents global distribution of the contact normal orientation in binary packings with $g=0.8$ and volume fraction varying from 0.2 to 0.8 . An increase in contribution of small particles in binary sample resulted in more disordered packing structure and more asymmetric distribution. In each sample, anisotropy in distribution of contact normal orientation with a favored vertical contact direction was observed. Contacts directed downward prevailed with contact direction close to $270^{\circ}$. A detailed analysis of results has shown that a percentage of these contacts in all contacts increased from $3.4 \%$ in packings with volume fraction of $0.2-6.2 \%$ in mixtures with $f=0.8$. In samples with $g=0.4$, percentage of contacts with contact direction close to $270^{\circ}$ in all contacts increased from 

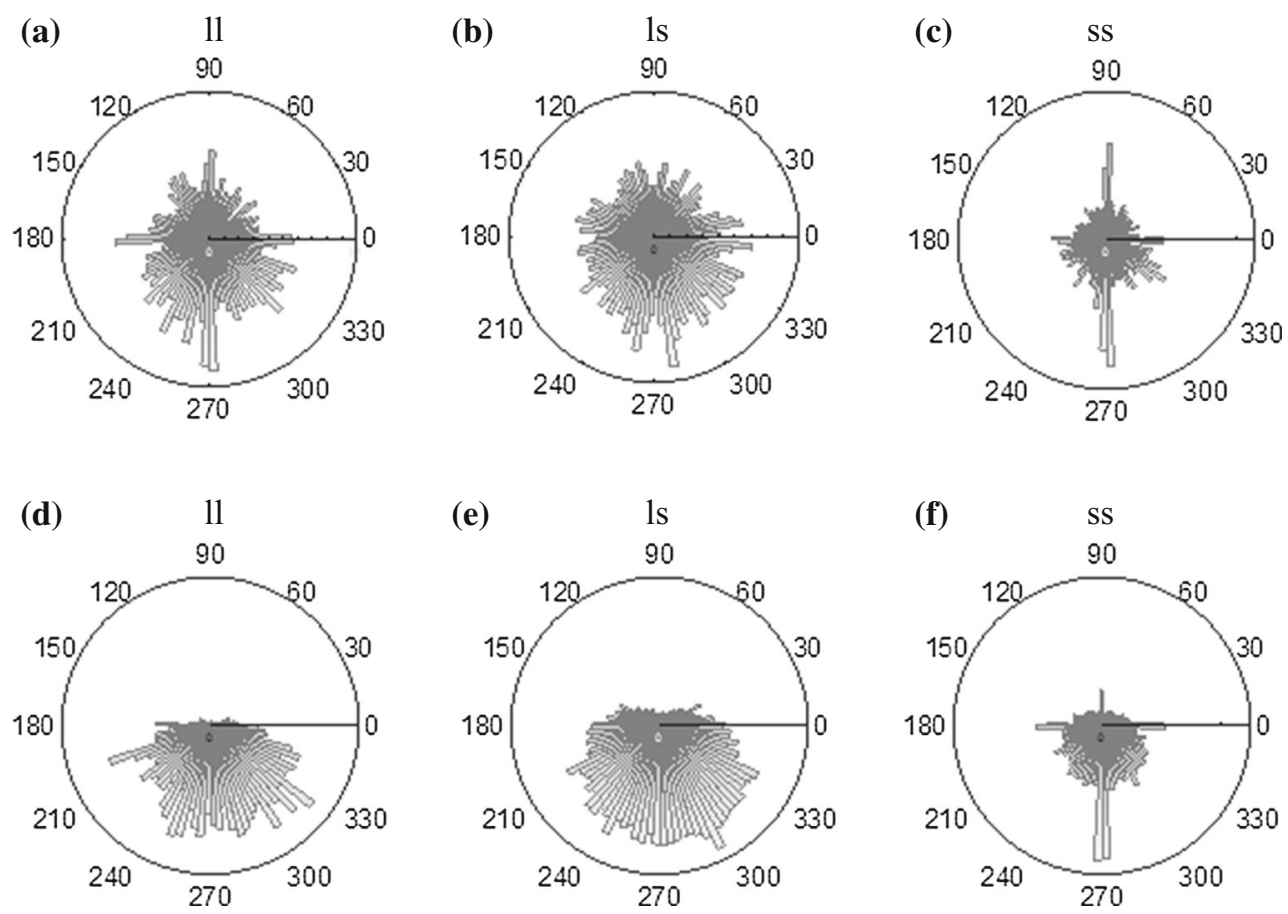

Fig. 3 Partial distributions of contact normal orientation in samples with $g=0.8(\mathbf{a}-\mathbf{c})$ and $g=0.4(\mathbf{d}-\mathbf{f})$, and volume fraction of small spheres $f=0.4$, under compressive load of $100 \mathrm{kPa}$

5.2 to $6 \%$ with $f$ value increasing from 0.2 to 0.8 . These results show an evident influence of contribution of granulometric fractions in binary granular packings on anisotropy of the contact normal orientation. However, that influence decreases with decreasing ratio of the diameter of small and large spheres. The discrepancies between findings presented in this paper and the ones reported by Sánchez et al. [9] result from larger differences between sizes of particles examined by Sánchez et al.

\subsection{Force and stress distribution}

The probability density functions of normal contact forces in samples with volume fraction of small spheres of 0.8 and various particle size ratios are presented in Fig. 5a. Distributions of normal contact forces are asymmetrical and left-skewed. The most homogeneous distribution of normal contact forces was obtained in sample with $g=0.8$. Probability density functions of normal contact forces narrowed in packings with decreasing ratio between small and large sphere. As the normal contact force is a function of the effective radius of contacting particles [1], the largest forces were obtained in samples with the smallest particle size ratio. Contribution of particle size fraction in an assembly was also found to have a strong influence on distribution of normal contact forces. Figure $5 \mathrm{~b}$ presents distributions of normal contact forces in mixtures with different $f$ values. Evolution of the packing structure of samples from ordered to disordered with an increase in contribution of small particles in mixtures up to $40 \%$ ( $f=0.4$ ) resulted in more heterogeneous distributions of normal contact forces and smaller average contact forces. A further increase in number of small particles in mixture resulted in more heterogeneous distribution of contact forces; however, no effect of composition of sample on the average contact force was observed in these samples. These results corroborate numerical findings of Wiacek and Molenda [17], who reported an increase in homogeneity of distributions of normal contact forces and a decrease in average contact forces with increasing degree of heterogeneity of polydisperse granular packing.

Distribution of contacts in the granular packing strongly influences transmission of forces and spatial distribution of force chains $[14,17]$. The studies of polydisperse packings, conducted by Voivret et al. [24] and Wiacek and Molenda [17] have shown that the strongest forces passed through the largest particles in system. Figure 6 presents an evolution of the average compressive force, defined as a sum of normal forces at contacts between particles, with volume fraction of small spheres in mixtures. The average compressive forces were normalized by mean compressive force in monodisperse sample comprising large particles. The forces exerted on spheres at contact points were found to be strictly related to the partial coordination numbers, presented by Wiacek [1]. The $\bar{F}$ values and coordination numbers for different contacts followed the same paths with increasing contribution of small particles in mixtures. The average compressive 

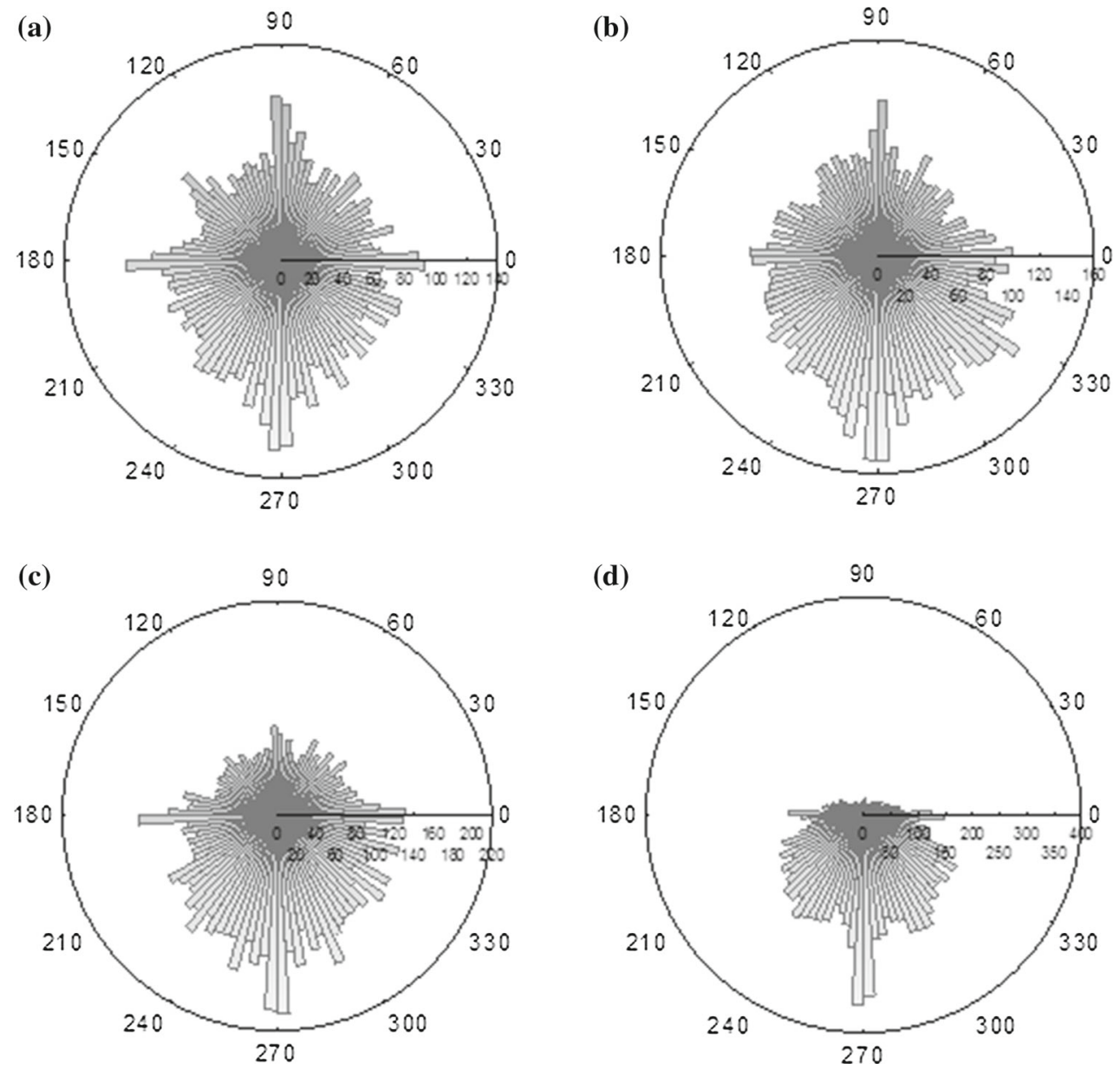

Fig. 4 Global distributions of contact normal orientation in mixtures with particle size ratio of 0.8 and volume fraction of small spheres of 0.2 (a), $0.4(\mathbf{b}), 0.6(\mathbf{c})$ and $0.8(\mathbf{e})$
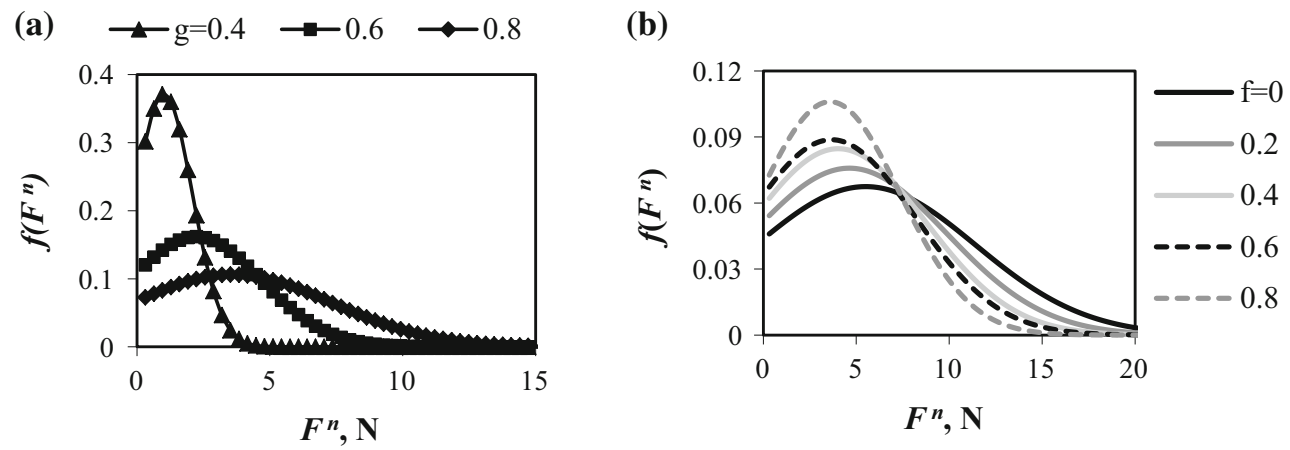

Fig. 5 Probability distribution functions of normal contact forces in samples with volume fraction of 0.8 and different size ratios (a) and size ratio of 0.8 and different volume fractions (b), when subjected to a vertical load of $100 \mathrm{kPa}$

forces exerted on small spheres by other particles were the largest in mixtures with $g$ value of 0.8 , which was strictly related to the largest number of contacts between these particles in mentioned samples [1]. The opposite tendency was observed for forces exerted on large spheres by small ones. The average compressive force exerted on large particles was higher in packings with smaller particle size ratio, wherein the largest average coordination number was observed [1]. Figure 6d shows that the average compressive force exerted on large spheres by the ones of the same size was slightly sensitive to the ratio of the diameter of small and large particles in binary mixture. The differences between $\bar{F}$ values in mixtures with different particle size ratio were small and they lied within the range of scatter. 


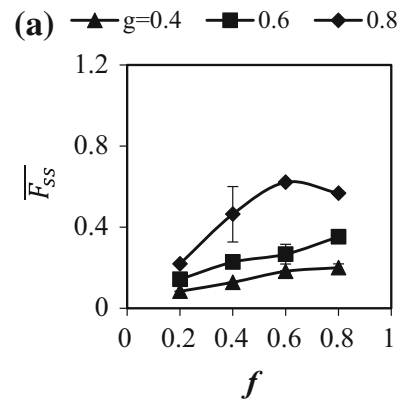

(c)

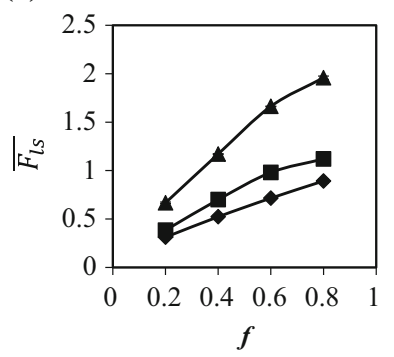

(b)

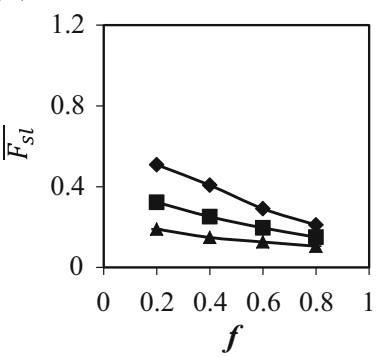

(d)

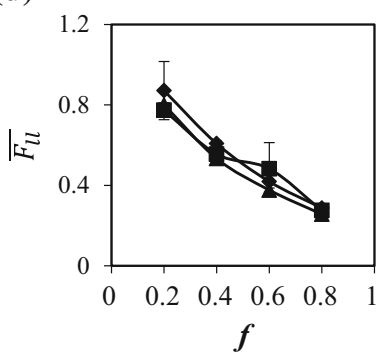

Fig. 6 Evolution of the average compressive forces originating from contacts between small particles (ss) (a), small and large particles (sl) (b), large and small particles (ls) (c) and large particles (11) (d) normalized by mean compressive force in monodisperse sample comprising large particles, with volume fraction of small particles

Regardless on the ratio of the diameter of small and large spheres and contribution of particle size fractions in binary mixtures, the largest compressive forces were exerted on the large spheres which confirms that mainly these particles contribute into stress transmission in granular bedding. Figure 7 shows the chains of compressive forces in compressed mixtures with $g=0.4$ and volume fraction of small spheres $f=0.2$ and $f=0.8$, in $x z$ plane. The black and white colors indicate the maximum and minimum values of forces.

The chains of the largest forces passed through the largest particles in samples, which has been already observed by Wiacek and Molenda [17] in polydisperse sphere packings.

In the binary granular packings, small spheres fill partially the voids between large grains and carry reduced effective stress. They may percolate through the primary fabric produced by immobile large particles $[25,26]$. Kenney and Lau [27] reported that the presence of primary fabric and loose small particles in particulate solid was an origin of internal instability of an assembly. The transfer of externally applied loads is not homogeneous in particulate bedding and stresses are transferred through limited number of particles. A number of studies have been performed over last few decades to measure the contribution of particle size fractions into stress transfer in granular materials $[14,28]$.

In this study, the effect of particle size ratio and volume fraction of small spheres on global and partial stress (by size particle) in bidisperse mixtures was investigated. The mean particle stress is defined as [29]:

$p^{p}=\frac{1}{3} \operatorname{tr}\left(\sigma^{p}\right)$

where the stress tensor components for a single particle are given by $[30,31]$ :

$\sigma_{i j}^{p}=\frac{1}{V_{p}} \sum_{c=1}^{N_{c}} l_{i}^{p c} F_{i j}^{n p c}$.

In Eq. (3), $V_{p}$ is a particle volume, $F_{i j}^{n p c}$ is a normal force exerted on particle $p$ at contact $c$ and $N_{c}$ is a number of contacts of particle $p$. The branch vector connecting the centre of the particle to its contact $\left(l_{i}^{p c}\right)$, associated with particle radius $R_{i}^{p}$ and displacement in normal direction $\delta_{n}^{c}$, is expressed as: (a)

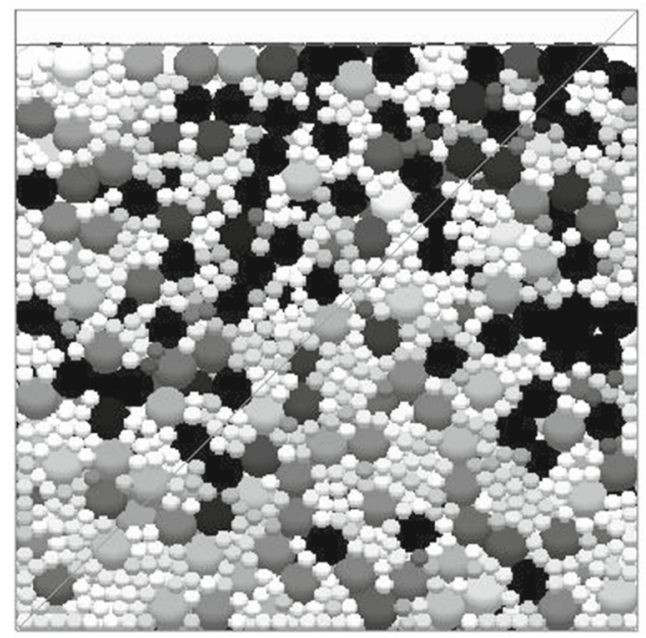

(b)

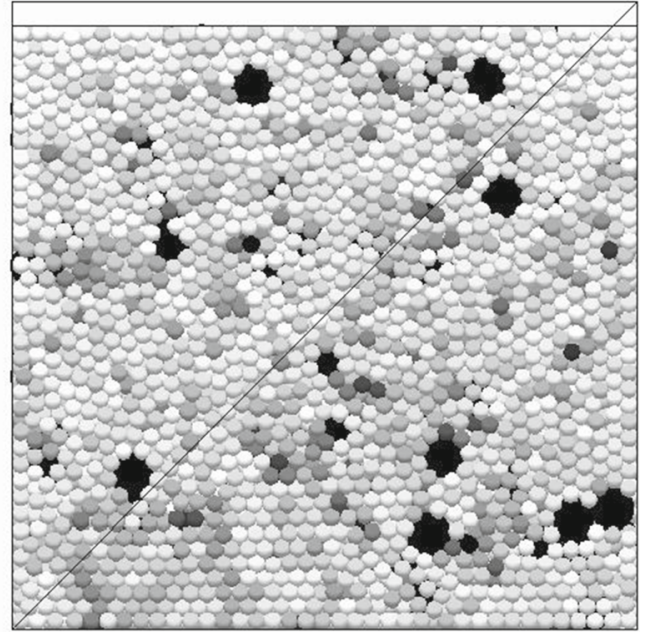

Fig. 7 Force chain networks in compressed mixtures with $g=0.8$ (a) and 0.4 (b), and volume fraction of small spheres of 0.2 


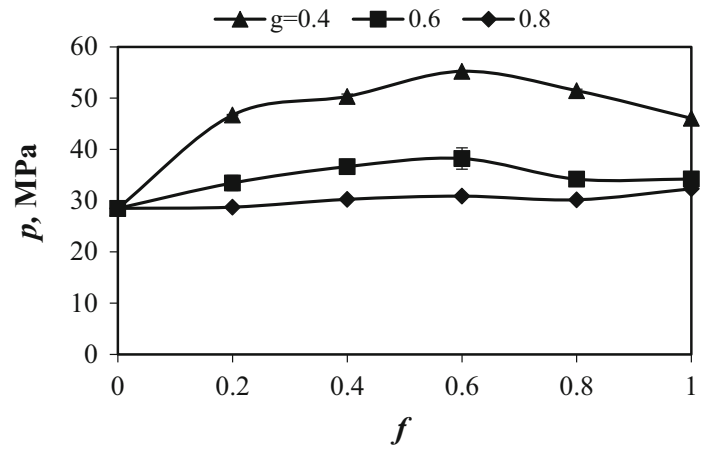

Fig. 8 The global stress in binary mixtures with various particle size ratios and volume fraction of small particles, when subjected to a vertical load of $100 \mathrm{kPa}$

$l_{i}^{p c}=\left(R_{i}^{p}-\frac{\delta_{n}^{c}}{2}\right)$

The mean normal stress for the whole sample comprising $N$ particles (global stress) is given by:

$p=\frac{1}{V} \sum_{p=1}^{N}\left(p^{p} V^{p}\right)$

where $V$ is a volume of sample. The mean normal stress for small spheres (partial stress) may be computed by:

$p_{s}=\frac{\Phi}{\sum_{p=1}^{N_{s}} V^{p}} \sum_{p=1}^{N_{s}}\left(p^{p} V^{p}\right)$

where $\Phi$ is a solid fraction of sample and $N_{s}$ is a number of small particles.

Figure 8 shows the evolution of global stress with volume fraction of small spheres in binary mixtures with different particle size ratios subjected to compressive load of $100 \mathrm{kPa}$. As the global stress in granular material is determined by its packing density, the $p(f)$ relationships were similar to the ones between solid fraction and volume fraction of small particles in sample. In samples with particle size ratio of 0.4 , the global stress increased twofold with volume fraction of small particles increasing form 0 to 0.6 due to increase in solid fraction of samples. A further increase in $f$ value decreased the mean normal stress. An increase in the ratio of the diameter of small and large spheres from 0.4 to 0.6 resulted in decrease in global stress by $30 \%$ in packings with volume fraction of small particles of 0.6. A slight relationship between global stress and contribution of small spheres was observed in samples with $g=0.8$, wherein slight changes in solid fraction and total compressive force with increasing $f$ value were also observed.

Figure 9 presents evolution of the ratio between pressure in small spheres and global pressure with contribution of small

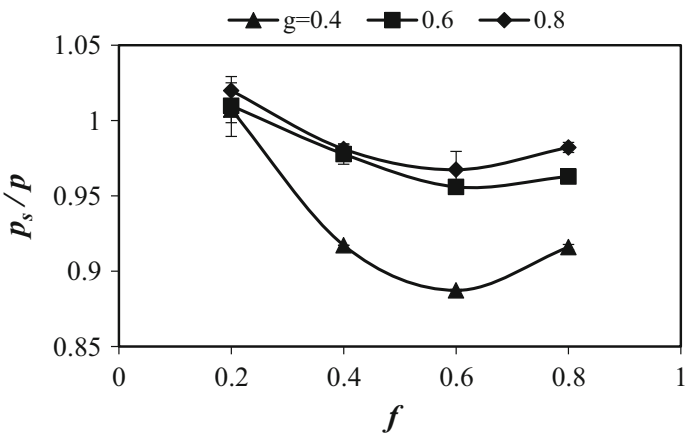

Fig. 9 Variation of stress distributed by small particles: to stress distributed by all particles in mixtures with various size ratios and volume fraction of small particles, for compressive load of $100 \mathrm{kPa}$

particles in samples. In packings with volume fraction of small particles of $20 \%(f=0.2)$, the $p_{s} / p \approx 1$, indicating that contribution of small and large spheres into stress transfer is approximately equal. A decrease in $p_{s} / p$ value with increasing volume fraction of small particles in mixtures up to 0.6 was observed, which was followed by slight increase in packings with higher $f$ values. In these samples, stress in small spheres was smaller than the global mean stress and it increased as the particle size ratio increased. At small $g$ values, small particles do not fit completely the voids between large ones and interact with the large grains to a lesser degree. Therefore, their contribution into stress transfer in granular packings was smaller. These results were partially consistent with the findings reported by Shire et al. [14] for binary packings with particle size ratios higher than 2 . The authors observed that small and large spheres contributed approximately equally into stress transfer in samples with volume fraction of small particles of 0.2 and stress in small particles decreased with decreasing ratio between diameter of small and large particles. No change in $p_{s} / p$ value with decreasing particle size ratio in packings with larger number of small particles was also observed by the authors. In this study, a strong influence of the contribution of particle size fractions in mixture on stress transfer in small components was found, which was due to small differences between diameters of spheres. In samples with size ratio larger than 0.4 , smaller spheres may not be tapered within the voids between larger particles which results in relationships between $p_{s} / p$ and volume contribution of particle size fractions different than the ones obtained for mixtures with larger particle size ratios.

\subsection{Energy dissipation}

The elastic energy accumulated at contact between two interacting spheres is defined as:

$E_{i j}=\int_{0}^{\delta} F_{i j} d \delta_{i j}$, 
where $F_{i j}$ is a contact force in normal or tangential direction and $\delta_{i j}$ is the normal or cumulative shear displacements [1]. For non-linear Hertz-Mindlin contact model, applied in this study, the elastic energies accumulated in the normal and tangential directions $\left(E_{i j}^{n}, E_{i j}^{t}\right)$ at contact between particles are given by:

$E_{i j}^{n}=\frac{2}{5} F_{i j}^{n} \delta_{i j}^{n}$

$E_{i j}^{n}=\frac{\left(F^{t}\right)^{2}}{2 k_{t}}$.

The sum of the above energies is a total elastic energy accumulated at the contact point between particles $(E)$.

It is well-known that the mechanical response of a granular packing subjected to external load is determined by the dissipative nature of material; however, the knowledge in that field is still insufficient. Therefore, in this study, the effect of the geometric and statistical factors on dissipation of energy in binary mixtures was investigated. The energy dissipated in granular packing is defined as:

$D=\Delta W-\Delta E$

where $\Delta W$ is the work done on the sample by external forces and $\Delta E$ is the change of total energy of the particulate assembly. The $\Delta W$ is calculated from the external force using the following Eq. [32]:

$\Delta W=\int_{0}^{\Delta H} F d H$,

where $F$ is the force applied on the top platen along the deformation direction and $\Delta \mathrm{H}$ is the displacement of the top platen.

Figure 10 illustrates evolution of the energy dissipation per contact in bidisperse packings with $g=0.8$ and different

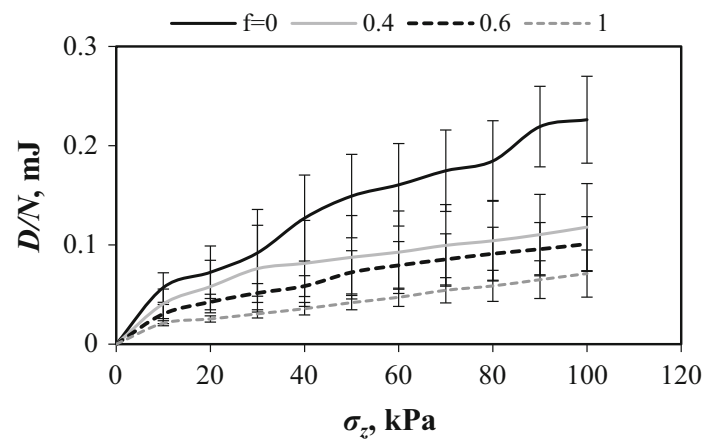

Fig. 10 Evolution of energy dissipation per number of contacts with volume fraction of small particles in mixtures with particle size ratio of 0.8 , when subjected to compressive load of $100 \mathrm{kPa}$

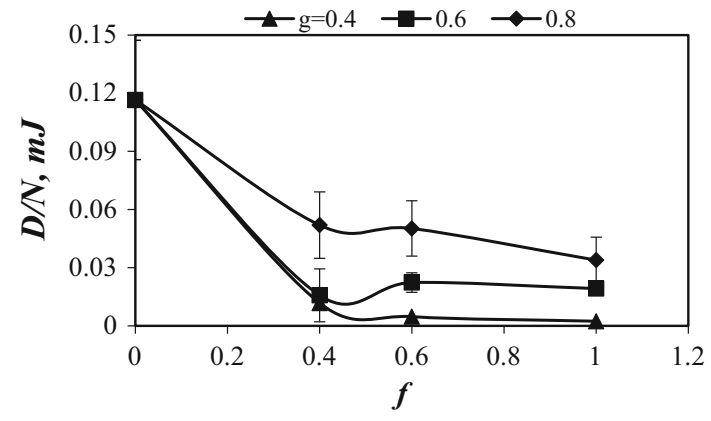

Fig. 11 Evolution of energy dissipation per number of contacts in mixtures with various size ratios and volume fractions of small particles, when subjected to compressive load of $100 \mathrm{kPa}$

volume fractions of small spheres, when subjected to compressive loads. Dissipation of energy increased with increasing vertical pressure, which was consistent with numerical results previously reported by Wiacek and Molenda [17]. Energy loss in the contact point between two particles is determined by contact force which is a function of the radii of the interacting spheres [1]. Therefore, a decrease in dissipation of energy with an increase in the contribution of small spheres in granular system was observed. For the same reason, energy dissipated in the contact points decreased with decreasing ratio of the diameter of small and large spheres in samples. The variation of energy dissipation per contact with increasing volume fraction of small particles in mixtures is presented in Fig. 11.

\section{Conclusions}

The micromechanics of the binary granular mixtures subjected to compressive load was investigated, using the 3D DEM simulations. The micromechanical properties of granular packing strongly determine the internal response of material to externally applied loads and its macromechanical properties; however, they alone are also dependent on few factors. Therefore, in this study, the analysis of the effect of the geometric and statistical factors on the micromechanics of binary sphere packings was conducted. The geometric factor was the ratio of the diameter of small and large spheres in samples, while the statistical one was a volume fraction of small spheres in mixture. The particle size ratio in bidisperse samples was chosen larger than 0.4 to prevent small particles from percolating through bedding.

Numerical investigations of the binary granular systems showed a strong influence of both, particle size ratio and volume fraction of small particles on distribution of normal contact forces in binary mixtures. The analysis of the compressive force, defined as a sum of normal 
forces at contacts between particles, indicated that partial compressive force and partial coordination numbers followed the same paths with increasing contribution of small particles in mixtures. In this study, a strong relationship between anisotropy of the contact normal orientation in bidisperse packings and geometric and statistical factors was also indicated. An increase in global anisotropy was observed with increasing difference between the dimeters of spheres in samples. An increase in volume fraction of small spheres in packings also resulted in higher anisotropy of the contact normal orientation, however, an increase in anisotropy was smaller in mixtures with smaller particle size ratios.

The global pressure and solid fraction have followed the same paths with increasing contribution of small particles in mixtures. In samples with the particle size ratio of 0.6 and 0.4 , solid fraction and global pressure reached maximum when the volume fraction of small spheres was 0.6 , which was not observed in samples with larger ratio of the diameter of small and large spheres. The distribution of stress in the particulate bedding was not homogeneous and it strongly depended on the composition of the mixture. In packings with volume fraction of small particles of $20 \%$, the contribution of small and large spheres into stress transfer was approximately equal. In mixtures with large number of small spheres and large particle size ratio, small spheres did not fit completely the voids between large ones and interacted with large grains to a lesser degree. Therefore, the stress in small spheres decreased as a volume fraction of small particles increased and ratio of the diameter of small and large spheres decreased. Both, the geometric and statistical factors were found to determine dissipation of energy in the examined packings. As the energy loss is a function of the radii of the interacting spheres, dissipation of energy decreased with decreasing particle size ratio and increasing volume fraction of small spheres in mixtures. These results indicate that the significant decrease in energy loss in particulate system may be achieved by selecting components of an appropriate size.

The bidisperse and multicomponent particulate packings are of great interest in many industrial processes and, therefore, an in-depth insight into the nature of these systems is required. The results presented in this study provide the detailed knowledge of micromechanical properties of binary granular mixtures, which may also pave the way for better understanding behavior of more complex packings of nonuniformly sized grains.

Acknowledgements This work was supported by the Ministry of Science and Higher Education of Poland [Grant Number 0625/IP2/2013/72]

\section{Compliance with ethical standards}

Conflict of interest The authors declare that they have no conflict of interest.
Open Access This article is distributed under the terms of the Creative Commons Attribution 4.0 International License (http://creativecomm ons.org/licenses/by/4.0/), which permits unrestricted use, distribution, and reproduction in any medium, provided you give appropriate credit to the original author(s) and the source, provide a link to the Creative Commons license, and indicate if changes were made.

\section{References}

1. Wiacek, J.: Geometrical parameters of binary granular mixtures with size ratio and volume fraction: experiments and DEM simulations. Granul. Matter 18, 42 (2016)

2. McGeary, R.K.: Mechanical packing of spherical particles. J. Am. Ceram. Soc. 44, 513-523 (1961)

3. Rassolusly, S.M.K.: The packing density of 'perfect' binary mixtures. Powder Technol. 103, 145-150 (1999)

4. Isola, R.: Packing of granular materials. Dissertation, University of Nottingham (2008)

5. Voivret, C., Radjaï, F., Delenne, J.-Y., El Youssoufi, M.S.: Spacefilling properties of polydisperse granular media. Phys. Rev. E 76, 021301 (2007)

6. Jalali, P., Li, M.: Model for estimation of critical packing density in polydisperse hard-disc packings. Phys. A 381, 230-238 (2007)

7. Robinson, D.A., Friedman, S.P.: Observations of the effects of particle shape and particle size distribution on avalanching of granular media. Phys. A 311, 97-110 (2002)

8. Martin, C.L., Bouvart, D.: Isostatic compaction of bimodal powder mixtures and composites. Int. J. Mech. Sci. 46, 907-927 (2004)

9. Sánchez, J., Auvient, G., Cambou, B.: Coordination number and geometric anisotropy in binary sphere mixtures. In: Soga, K., Kumar, K., Biscontin, G., Kuo, M. (eds.) Geomechanics from Micro to Macro. Taylor \& Francis Group, London (2004)

10. Skrinjar, O., Larsson, P.-L.: On discrete element modelling of compaction of powders with size ratio. Comput. Mater. Sci. 31, 131-146 (2004)

11. Desmond, K.W., Weeks, E.R.: Influence of particle size distribution on random close packing of spheres. Phys. Rev. E 90, 022204 (2014)

12. Pinson, D., Zou, R.P., Yu, A.B., Zulli, P., McCarthy, M.J.: Coordination number of binary mixtures of spheres. J. Phys. D 31, 457-462 (1998)

13. Lade, P.V., Liggio, C.D., Yamamuro, J.A.: Effects of non-plastic fines on minimum and maximum void ratios of sand. Geotech. Test. J. 21, 336-347 (1998)

14. Shire, T., O'Sullivan, C., Hanley, K.: The influence of finer fraction and size-ratio on the micro-scale properties of dense bimodal materials. In: Soga, K., Kumar, K., Biscontin, G., Kuo, M. (eds.) Geomechanics from Micro to Macro. Taylor \& Francis Group, London (2015)

15. Zhao, Z., Liu, C., Brogliato, V.: Energy dissipation and dispersion effects in granular media. Phys. Rev. E 78, 031307 (2008)

16. Nguyen, N.-S., Zhang, H., Brogliato, B.: Multiple impacts with friction in the rocking block and tapered chains. In: Bernardini, D., Rega, G., Romeo, F. (eds.) Proceedings of 7th European Nonlinear Dynamics Conference-ENOC 2011, Rome (2011)

17. Wiącek, J., Molenda, M.: Effect of particle polydsipersity on micromechanical properties and energy dissipation in granular mixtures. Particuology 16, 91-99 (2014)

18. Karion, A., Hunt, M.L.: Energy dissipation in sheared granular flows. J. Heat Transf. 121, 984-991 (1999)

19. Wiącek, J., Molenda, M.: Effect of particle size distribution on micro- and macromechanical response of granular packings under compression. Int. J. Solids Struct. 51, 4189-4195 (2014) 
20. Cundall, P.A., Strack, O.D.: A discrete element model for granular assemblies. Géotechnique 29, 47-65 (1979)

21. EDEM Software. Retrieved from www.dem-solutions.com/ software/edem-software

22. Wiącek, J., Molenda, M.: Representative elementary volume analysis of polydisperse granular packings using discrete element method. Particuology 27, 88-94 (2016)

23. Eurocode 1: Actions on structures. Part 4. Silos and tanks, EN 1991-4 (2006)

24. Voivret, C., Radjaï, F., Delenne, J.Y., El Youssoufi, M.S.: Multiscale force networks in highly polydisperse granular media. Phys. Rev. Lett. 102, 188001 (2009)

25. To, H.D., Galindo-Torres, S.A., Scheuermann, A.: A numerical approach for the determination of the primary fabric of granular soils. Appl. Mech. Mater. 553, 489-494 (2014)

26. To, H.D., Galindo-Torres, S.A., Scheuermann, A.: Primary fabric fraction analysis of granular soils. Acta Geotech. 10, 375-387 (2015)
27. Kenney, T., Lau, D.: Internal stability of granular filters. Can. Geotech. J. 22, 215-225 (1985)

28. Shire, T., O’Sullivan, C., Hanley, K., Fannin, R.: Fabric and effective stress distribution in internally unstable soils. J. Geotech. Geoenviron. Eng. 140, 04014072 (2014)

29. Andrade, J.E., Avila, C.F., Hall, S.A., Lenoir, N., Viggiani, G.: Multiscale modeling and characterization of granular matter: from grain kinematics to continuum mechanics. J. Mech. Phys. Solids 59, 237-250 (2011)

30. Durán, O., Kruyt, N.P., Luding, S.: Analysis of three-dimensional micro-mechanical strain formulations of granular materials: Evoluation of accuracy. Int. J. Solids Struct. 47, 251-260 (2010)

31. Göncü, F., Durán, O., Luding, S.: Constitutive relations for the isotropic deformation of frictionless packings of polydisperse spheres. C.R. Mec. 338, 570-586 (2010)

32. Yang, F., Zhong, Z.: On the energy conservation during the active deformation in molecular dynamics simulations. J. Mech. Phys. Solids 77, 146-157 (2015) 